\title{
Periodic Solutions of Some Polynomial Differential Systems in Dimension 3 via Averaging Theory
}

\author{
Amar Makhlouf and Lilia Bousbiat \\ Laboratory LMA, Department of Mathematics, University of Annaba, Elhadjar, 23 Annaba, Algeria \\ Correspondence should be addressed to Amar Makhlouf; makhloufamar@yahoo.fr \\ Received 5 July 2015; Accepted 27 August 2015 \\ Academic Editor: Jaume Giné
}

Copyright ( 2015 A. Makhlouf and L. Bousbiat. This is an open access article distributed under the Creative Commons Attribution License, which permits unrestricted use, distribution, and reproduction in any medium, provided the original work is properly cited.

We provide sufficient conditions for the existence of periodic solutions of the polynomial third order differential system $\dot{x}=-y+$ $\varepsilon P(x, y, z)+h_{1}(t), \dot{y}=x+\varepsilon Q(x, y, z)+h_{2}(t)$, and $\dot{z}=a z+\varepsilon R(x, y, z)+h_{3}(t)$, where $P, Q$, and $R$ are polynomials in the variables $x, y$, and $z$ of degree $n, h_{i}(t)=h_{i}(t+2 \pi)$ with $i=1,2,3$ being periodic functions, $a$ is a real number, and $\varepsilon$ is a small parameter.

\section{Introduction and Statements of Results}

One of the main problems in the theory of differential systems is the study of their periodic orbits, their existence, their number, and their stability. As usual, a limit cycle of a differential equation is a periodic orbit isolated in the set of all periodic orbits of the differential equation.

In this paper, we study the existence of the periodic orbits of the polynomial third order differential system

$$
\begin{aligned}
& \dot{x}=-y+\varepsilon P(x, y, z)+h_{1}(t), \\
& \dot{y}=x+\varepsilon Q(x, y, z)+h_{2}(t), \\
& \dot{z}=a z+\varepsilon R(x, y, z)+h_{3}(t),
\end{aligned}
$$

where $a$ is a real number, $P, Q$, and $R$ are polynomials in the variables $x, y$, and $z$ of degree $n, h_{i}(t)=h_{i}(t+2 \pi)$ with $i=1,2,3$ being periodic functions, and $\varepsilon$ is a small parameter.

This problem has been studied in the homogeneous case (more precisely in the case where $h_{i}(t)=0$ with $i=$ $1,2,3)$ by different authors by applying other versions of theorems of averaging method; see for instance [1-4]. More precisely, in paper [1], the authors considered the following system:

$$
\begin{aligned}
& \dot{x}=-y+\varepsilon P(x, y, z), \\
& \dot{y}=x+\varepsilon Q(x, y, z), \\
& \dot{z}=\varepsilon R(x, y, z),
\end{aligned}
$$

where $P, Q$, and $R$ are polynomials in the variables $x, y$, and $z$ of degree $n$. They found that this system has at most $n(n-1) / 2$ limit cycles bifurcating from the periodic orbits of the linear system $\dot{x}=-y, \dot{y}=x$, and $\dot{z}=0$. Moreover, there are such perturbed systems having $n(n-1) / 2$ limit cycles. In paper [2], the authors considered the following system:

$$
\begin{aligned}
& \dot{x}=-y+\varepsilon(a x+P(x, y, z)), \\
& \dot{y}=x+\varepsilon(a y+Q(x, y, z)), \\
& \dot{z}=\varepsilon(c z+R(x, y, z)),
\end{aligned}
$$

where $P, Q$, and $R$ are arbitrary polynomials of degree $n$ starting with terms of degree 2 . They found that there are systems in the previous system having at least $n(n-1) / 2$ limit cycles bifurcating from the periodic orbits of the system 
$\dot{x}=-y, \dot{y}=x$, and $\dot{z}=0$. In paper [3], the authors considered the following differential system:

$$
\begin{aligned}
& \dot{x}=-y+\varepsilon P(x, y, z), \\
& \dot{y}=x+\varepsilon Q(x, y, z)+\varepsilon \cos t, \\
& \dot{z}=a z+\varepsilon R(x, y, z)
\end{aligned}
$$

where $P, Q$, and $R$ are polynomials in the variables $x, y$, and $z$ of degree $n$ and $a$ is a real number different from zero. They found that this system has at least $m \in\{1,2, \ldots, 2[(n-$ $1) / 2]+1\}$ limit cycles bifurcating from the periodic orbits of the linear center contained in $z=0$ when $\varepsilon=0$. In [4], the authors considered the homogeneous case of system (1) with $a=0$ and the polynomials $P, Q$, and $R$ of degrees $n_{1}, n_{2}$, and $n_{3}$. They found that there are at most $[(m-1) / 2] n_{3}$ limit cycles bifurcating from the periodic orbits of the linear differential system $\dot{x}=-y, \dot{y}=x$, and $\dot{z}=0$ where $m=\max \left\{n_{1}, n_{2}\right\}$. In [5-8], the authors studied the limit cycles of classes of three order differential equations using averaging theory. There have been many papers studying the periodic solutions to third-order differential equations using Schauder's or Leray Schauder's fixed point theorem (see [9-11]) or the nonlocal reduction method (see $[12,13]$ ).

There are very few results which study the bifurcation of limit cycle from the periodic orbits for 3-dimensional systems in $\mathbb{R}^{3}$; see $[14,15]$.

To obtain analytically periodic solutions is in general a very difficult work, usually impossible. Here with the averaging theory we reduce this difficult problem for differential system (1) to find the zeros of a nonlinear system of three equations with three unknowns. It is known that in general the averaging theory for finding periodic solutions does not provide all the periodic solutions of the system. To explain this idea, there are two main reasons. First, the averaging theory for studying the periodic solutions of a differential system is based on the so-called displacement function, whose zeros provide periodic solutions. This displacement function in general is not global and consequently it cannot control all the periodic solution, only the ones which are in its domain of definition and that are hyperbolic. Second, the displacement function is expanded in power series of a small parameter $\varepsilon$, and the averaging theory only controls the zeros of the dominant term of this displacement function. When the dominant term is $\varepsilon^{k}$, we talk about the averaging theory of order $k$. For more details, see for instance [16] and the references quoted there. For more information about averaging theory, see Section 2 of this paper.

Our main results on the periodic solutions of the differential system (1) are the following ones.

Theorem 1. One considers differential system (1) with $a=0$. One defines

$$
\begin{aligned}
& \mathscr{F}_{1}\left(x_{0}, y_{0}, z_{0}\right)=\frac{1}{2 \pi} \int_{0}^{2 \pi}(\cos (t) P(A(t), B(t), C(t)) \\
& \quad+\sin (t) Q(A(t), B(t), C(t))) d t,
\end{aligned}
$$

$$
\begin{aligned}
& \mathscr{F}_{2}\left(x_{0}, y_{0}, z_{0}\right)=\frac{1}{2 \pi} \\
& \quad \cdot \int_{0}^{2 \pi}(-\sin (t) P(A(t), B(t), C(t)) \\
& +\cos (t) Q(A(t), B(t), C(t))) d t, \\
& \mathscr{F}_{3}\left(x_{0}, y_{0}, z_{0}\right)=\frac{1}{2 \pi} \int_{0}^{2 \pi} R(A(t), B(t), C(t)) d t,
\end{aligned}
$$

where

$$
\begin{aligned}
A(t)= & \cos (t) x_{0}-\sin (t) y_{0} \\
& +\int_{0}^{t}\left(\cos (t-s) h_{1}(s)-\sin (t-s) h_{2}(s)\right) d s, \\
B(t)= & \sin (t) x_{0}+\cos (t) y_{0} \\
& +\int_{0}^{t}\left(\sin (t-s) h_{1}(s)+\cos (t-s) h_{2}(s)\right) d s, \\
C(t)= & z_{0}+\int_{0}^{t} h_{3}(s) d s .
\end{aligned}
$$

If

$$
\begin{array}{r}
\int_{0}^{2 \pi}\left(\cos (s) h_{1}(s)-\sin (s) h_{2}(s)\right) d s=0 \\
\int_{0}^{2 \pi}\left(-\sin (s) h_{1}(s)+\cos (s) h_{2}(s)\right) d s=0 \\
\int_{0}^{2 \pi} h_{3}(s) d s=0
\end{array}
$$

then for every $\left(x_{0}^{*}, y_{0}^{*}, z_{0}^{*}\right)$ solution of the system

$$
\mathscr{F}_{k}\left(x_{0}, y_{0}, z_{0}\right)=0, \quad k=1,2,3,
$$

satisfying

$$
\operatorname{det}\left(\left.\frac{\partial\left(\mathscr{F}_{1}, \mathscr{F}_{2}, \mathscr{F}_{3}\right)}{\partial\left(x_{0}, y_{0}, z_{0}\right)}\right|_{\left(x_{0}, y_{0}, z_{0}\right)=\left(x_{0}^{*}, y_{0}^{*}, z_{0}^{*}\right)}\right) \neq 0,
$$

differential system (1) with $a=0$ has a periodic solution $\left(\begin{array}{l}x(t, \varepsilon) \\ y(t, \varepsilon) \\ z(t, \varepsilon)\end{array}\right)$, which tends to the periodic solution given by 


$$
\left(\begin{array}{c}
x(t) \\
y(t) \\
z(t)
\end{array}\right)=\left(\begin{array}{c}
\cos (t) x_{0}^{*}-\sin (t) y_{0}^{*}+\int_{0}^{t}\left(\cos (t-s) h_{1}(s)-\sin (t-s) h_{2}(s)\right) d s \\
\sin (t) x_{0}^{*}+\cos (t) y_{0}^{*}+\int_{0}^{t}\left(\sin (t-s) h_{1}(s)+\cos (t-s) h_{2}(s)\right) d s \\
z_{0}^{*}+\int_{0}^{t} h_{3}(s) d s
\end{array}\right)
$$

of the differential system

$$
\begin{aligned}
& \dot{x}=-y+h_{1}(t), \\
& \dot{y}=x+h_{2}(t), \\
& \dot{z}=h_{3}(t),
\end{aligned}
$$

$$
\begin{aligned}
C(t)= & \frac{e^{a t}}{1-e^{2 \pi a}} \int_{0}^{2 \pi} e^{a(2 \pi-s)} h_{3}(s) d s \\
& +\int_{0}^{t} e^{a(t-s)} h_{3}(s) d s .
\end{aligned}
$$

when $\varepsilon \rightarrow 0$.

Note that this solution is periodic of period $2 \pi$.

Theorem 2. One considers differential system (1) with $a \neq 0$. One defines

$$
\begin{aligned}
& \mathscr{F}_{1}\left(x_{0}, y_{0}\right)=\frac{1}{2 \pi} \int_{0}^{2 \pi}(\cos (t) P(A(t), B(t), C(t)) \\
& +\sin (t) Q(A(t), B(t), C(t))) d t \\
& \mathscr{F}_{2}\left(x_{0}, y_{0}\right)=\frac{1}{2 \pi} \int_{0}^{2 \pi}(-\sin (t) P(A(t), B(t), C(t)) \\
& \quad+\cos (t) Q(A(t), B(t), C(t))) d t
\end{aligned}
$$

where

$$
\begin{aligned}
A(t)= & \cos (t) x_{0}-\sin (t) y_{0} \\
& +\int_{0}^{t}\left(\cos (t-s) h_{1}(s)-\sin (t-s) h_{2}(s)\right) d s, \\
B(t)= & \sin (t) x_{0}+\cos (t) y_{0} \\
& +\int_{0}^{t}\left(\sin (t-s) h_{1}(s)+\cos (t-s) h_{2}(s)\right) d s,
\end{aligned}
$$

$$
\begin{aligned}
& \int_{0}^{2 \pi}\left(\cos (s) h_{1}(s)+\sin (s) h_{2}(s)\right) d s=0, \\
& \int_{0}^{2 \pi}\left(-\sin (s) h_{1}(s)+\cos (s) h_{2}(s)\right) d s=0, \\
& z_{0}=\frac{1}{1-e^{2 \pi a}} \int_{0}^{2 \pi} e^{a(2 \pi-s)} h_{3}(s) d s,
\end{aligned}
$$

If

then for every $\left(x_{0}^{*}, y_{0}^{*}\right)$ solution of the system

$$
\mathscr{F}_{k}\left(x_{0}, y_{0}\right)=0, \quad k=1,2 \text {, }
$$

satisfying

$$
\operatorname{det}\left(\left.\frac{\partial\left(\mathscr{F}_{1}, \mathscr{F}_{2}\right)}{\partial\left(x_{0}, y_{0}\right)}\right|_{\left(x_{0}, y_{0}\right)=\left(x_{0}^{*}, y_{0}^{*}\right)}\right) \neq 0,
$$

differential system (1) with $a \neq 0$ has a periodic solution $\left(\begin{array}{l}x(t, \varepsilon) \\ y(t, \varepsilon) \\ z(t, \varepsilon)\end{array}\right)$, which tends to the periodic solution given by

$$
\left(\begin{array}{c}
x(t) \\
y(t) \\
z(t)
\end{array}\right)=\left(\begin{array}{c}
\cos (t) x_{0}^{*}-\sin (t) y_{0}^{*}+\int_{0}^{t}\left(\cos (t-s) h_{1}(s)-\sin (t-s) h_{2}(s)\right) d s \\
\sin (t) x_{0}^{*}+\cos (t) y_{0}^{*}+\int_{0}^{t} \sin (t-s) h_{1}(s)+\cos (t-s) h_{2}(s) d s \\
\frac{e^{a t}}{1-e^{2 \pi a}} \int_{0}^{2 \pi} e^{a(2 \pi-s)} h_{3}(s) d s+\int_{0}^{t} e^{a(t-s)} h_{3}(s) d s
\end{array}\right)
$$


of the differential system

$$
\begin{aligned}
& \dot{x}=-y+h_{1}(t), \\
& \dot{y}=x+h_{2}(t), \\
& \dot{z}=a z+h_{3}(t),
\end{aligned}
$$

when $\varepsilon \rightarrow 0$.

Note that this solution is periodic of period $2 \pi$.

Theorems 1 and 2 are proved in Section 3. Their proofs are based on the averaging theory for computing periodic orbits; see Section 2.

Corollary 3. Consider differential system (1) with $a=0$ where

$$
\begin{aligned}
\left(\begin{array}{l}
h_{1}(t) \\
h_{2}(t) \\
h_{3}(t)
\end{array}\right) & =\left(\begin{array}{c}
\sin (t) \\
\cos (t) \\
-\sin (t)
\end{array}\right), \\
\left(\begin{array}{l}
P(x, y, z) \\
Q(x, y, z) \\
R(x, y, z)
\end{array}\right) & =\left(\begin{array}{c}
x^{2}+y^{2}+x y z \\
y^{2}+z^{2}+x y z \\
x+y+z
\end{array}\right) .
\end{aligned}
$$

Then, this differential system has four periodic solutions $\left(\begin{array}{l}x_{k}(t, \varepsilon) \\ y_{k}(t, \varepsilon) \\ z_{k}(t, \varepsilon)\end{array}\right)$ with $k=1,2,3,4$, tending to the periodic solutions $\left(\begin{array}{l}x_{k}(t) \\ y_{k}(t) \\ z_{k}(t)\end{array}\right)$ where

$$
\begin{aligned}
& \left(\begin{array}{l}
x_{1}(t) \\
y_{1}(t) \\
z_{1}(t)
\end{array}\right)=\left(\begin{array}{c}
0 \\
\sin (t) \\
\cos (t)
\end{array}\right) \\
& \left(\begin{array}{l}
x_{2}(t) \\
y_{2}(t) \\
z_{2}(t)
\end{array}\right)=\left(\begin{array}{c}
-\cos (t) \\
0 \\
\cos (t)
\end{array}\right) \\
& \left(\begin{array}{l}
x_{3}(t) \\
y_{3}(t) \\
z_{3}(t)
\end{array}\right)=\left(\begin{array}{c}
\frac{1}{2} \cos (t)-\frac{\sqrt{3}}{2} \sin (t) \\
\frac{3}{2} \sin (t)+\frac{\sqrt{3}}{2} \cos (t) \\
\cos (t)
\end{array}\right) \\
& \left(\begin{array}{l}
x_{4}(t) \\
y_{4}(t) \\
z_{4}(t)
\end{array}\right)=\left(\begin{array}{c}
\frac{1}{2} \cos (t)+\frac{\sqrt{3}}{2} \sin (t) \\
\frac{3}{2} \sin (t)-\frac{\sqrt{3}}{2} \cos (t)
\end{array}\right)
\end{aligned}
$$

of the differential system

$$
\begin{aligned}
& \dot{x}=-y+\sin (t), \\
& \dot{y}=x+\cos (t), \\
& \dot{z}=-\sin (t),
\end{aligned}
$$

when $\varepsilon \rightarrow 0$.

Corollary 3 is proved in Section 3.
Corollary 4. Consider differential system (1) with $a \neq 0$ where

$$
\begin{aligned}
\left(\begin{array}{l}
h_{1}(t) \\
h_{2}(t) \\
h_{3}(t)
\end{array}\right) & =\left(\begin{array}{c}
\sin (t) \\
\cos (t) \\
\cos (t)
\end{array}\right), \\
\left(\begin{array}{l}
P(x, y, z) \\
Q(x, y, z) \\
R(x, y, z)
\end{array}\right) & =\left(\begin{array}{c}
x+y-x^{2} y \\
x+y-x y^{2} \\
x+y+z
\end{array}\right) .
\end{aligned}
$$

Then, this differential system has two periodic solutions $\left(\begin{array}{l}x_{1}(t, \varepsilon) \\ y_{1}(t, \varepsilon) \\ z_{1}(t, \varepsilon)\end{array}\right),\left(\begin{array}{l}x_{2}(t, \varepsilon) \\ y_{2}(t, \varepsilon) \\ z_{2}(t, \varepsilon)\end{array}\right)$ tending to the periodic solutions

$$
\begin{aligned}
& \left(\begin{array}{l}
x_{1}(t) \\
y_{1}(t) \\
z_{1}(t)
\end{array}\right) \\
& =\left(\begin{array}{c}
-\frac{1}{2} \cos (t)+\left(\frac{4}{5}-\frac{\sqrt{139}}{10}\right) \sin (t) \\
\frac{1}{2} \sin (t)+\left(-\frac{4}{5}+\frac{\sqrt{139}}{10}\right) \cos (t) \\
\frac{-a \cos (t)+\sin (t)}{a^{2}+1} \\
y_{2}(t) \\
z_{2}(t)
\end{array}\right), \\
& =\left(\begin{array}{c}
x_{2} \\
-\frac{1}{2} \cos (t)+\left(\frac{4}{5}+\frac{\sqrt{139}}{10}\right) \sin (t) \\
\frac{1}{2} \sin (t)-\left(\frac{4}{5}+\frac{\sqrt{139}}{10}\right) \cos (t) \\
\frac{-a \cos (t)+\sin (t)}{a^{2}+1}
\end{array}\right)
\end{aligned}
$$

of the differential system

$$
\begin{aligned}
& \dot{x}=-y+\sin (t), \\
& \dot{y}=x+\cos (t), \\
& \dot{z}=a z+\cos (t),
\end{aligned}
$$

when $\varepsilon \rightarrow 0$.

Corollary 4 is proved in Section 3.

\section{Basic Results on Averaging Theory}

In this section, we present the basic results on the averaging theory that we will need for proving the main results of this paper.

We consider the problem of the bifurcation of $T$-periodic solutions from differential systems of the form

$$
\dot{\mathbf{x}}=F_{0}(t, \mathbf{x})+\varepsilon F_{1}(t, \mathbf{x})+\varepsilon^{2} F_{2}(t, \mathbf{x}, \varepsilon)
$$


with $\varepsilon=0$ to $\varepsilon \neq 0$ being sufficiently small. Here the functions $F_{0}, F_{1}: \mathbb{R} \times \Omega \rightarrow \mathbb{R}^{n}$ and $F_{2}: \mathbb{R} \times \Omega \times\left(-\varepsilon_{0}, \varepsilon_{0}\right) \rightarrow \mathbb{R}^{n}$ are $C^{2}$ functions, $T$-periodic in the first variable, and $\Omega$ is an open subset of $\mathbb{R}^{n}$. The main assumption is that the unperturbed system

$$
\dot{\mathbf{x}}=F_{0}(t, \mathbf{x})
$$

has a submanifold of periodic solutions. A solution of this problem is given using the averaging theory. For a general introduction to the averaging theory, see the books of Sanders and Verhulst [17] and of Verhulst [18].

Let $\mathbf{x}(t, \mathbf{z}, \varepsilon)$ be the solution of system (26) such that $\mathbf{x}(0, \mathbf{z}, \varepsilon)=\mathbf{z}$. We write the linearization of the unperturbed system along a periodic solution $\mathbf{x}(t, \mathbf{z}, 0)$ as

$$
\dot{\mathbf{y}}=D_{\mathbf{x}} F_{0}(t, \mathbf{x}(t, \mathbf{z}, 0)) \mathbf{y}
$$

In what follows we denote by $M_{\mathbf{z}}(t)$ some fundamental matrix of linear differential system (27) and by $\xi: \mathbb{R}^{k} \times$ $\mathbb{R}^{n-k} \rightarrow \mathbb{R}^{k}$ the projection of $\mathbb{R}^{n}$ onto its first $k$ coordinates; that is, $\xi\left(x_{1}, \ldots, x_{n}\right)=\left(x_{1}, \ldots, x_{k}\right)$. We assume that there exists a $k$-dimensional submanifold $\mathbf{Z}$ of $\Omega$ filled with $T$ periodic solutions of (26). Then, an answer to the problem of bifurcation of $T$-periodic solutions from the periodic solutions contained in $\mathbf{Z}$ for system (25) is given in the following result.

Theorem 5. Let $W$ be an open and bounded subset of $\mathbb{R}^{k}$, and let $\beta: \mathrm{Cl}(W) \rightarrow \mathbb{R}^{n-k}$ be a $C^{2}$ function. We assume that

(i) $\mathbf{Z}=\left\{\mathbf{z}_{\alpha}=(\alpha, \beta(\alpha)), \alpha \in \mathrm{Cl}(W)\right\} \subset \Omega$ and that for each $\mathbf{z}_{\alpha} \in \mathbf{Z}$ the solution $\mathbf{x}\left(t, \mathbf{z}_{\alpha}\right)$ of (26) is T-periodic;

(ii) for each $\mathbf{z}_{\alpha} \in \mathbf{Z}$ there is a fundamental matrix $M_{\mathbf{z}_{\alpha}}(t)$ of (27) such that the matrix $M_{\mathbf{z}_{\alpha}}^{-1}(0)-M_{\mathbf{z}_{\alpha}}^{-1}(T)$ has in the upper right corner the $k \times(n-k)$ zero matrix and in the lower right corner $a(n-k) \times(n-k)$ matrix $\Delta_{\alpha}$ with $\operatorname{det}\left(\Delta_{\alpha}\right) \neq 0$.

We consider the function $\mathscr{F}: \mathrm{Cl}(W) \rightarrow \mathbb{R}^{k}:$

$$
\mathscr{F}(\boldsymbol{\alpha})=\xi\left(\frac{1}{T} \int_{0}^{T} M_{\mathbf{z}_{\alpha}}^{-1}(t) F_{1}\left(t, \mathbf{x}\left(t, \mathbf{z}_{\alpha}\right)\right) d t\right)
$$

If there exists $a \in W$ with $\mathscr{F}(a)=0$ and $\operatorname{det}((d \mathscr{F})$ $d \alpha)(a)) \neq 0$, then there is a $T$-periodic solution $\varphi(t, \varepsilon)$ of system (25) such that $\varphi(0, \varepsilon) \rightarrow \mathbf{z}_{a}$ as $\varepsilon \rightarrow 0$.

Theorem 5 goes back to Malkin [19] and Roseau [20]; for a shorter proof see [21].

We assume that there exists an open set $V$ with $\mathrm{Cl}(V) \subset \Omega$ such that, for each $\mathbf{z} \in \mathrm{Cl}(V), \mathbf{x}(t, \mathbf{z}, 0)$ is $T$-periodic, where $\mathbf{x}(t, \mathbf{z}, 0)$ denotes the solution of the unperturbed system (26) with $\mathbf{x}(0, \mathbf{z}, 0)=\mathbf{z}$. The set $\mathrm{Cl}(V)$ is isochronous for system (25); that is, it is a set formed only by periodic orbits, all of them having the same period. Then, an answer to the problem of the bifurcation of $T$-periodic solutions from the periodic solutions $\mathbf{x}(t, \mathbf{z}, 0)$ contained in $\mathrm{Cl}(V)$ is given in the following result.

Theorem 6 (perturbations of an isochronous set). One assumes that there exists an open and bounded set $V$ with $\mathrm{Cl}(V) \subset \Omega$ such that, for each $\mathbf{z} \in \mathrm{Cl}(V)$, the solution $\mathbf{x}(t, \mathbf{z}, 0)$ is T-periodic, considering a function $\mathscr{F}: \mathrm{Cl}(V) \rightarrow \mathbb{R}^{n}$ defined by

$$
\mathscr{F}(\mathbf{z})=\int_{0}^{T} M_{\mathbf{z}}^{-1}(t) F_{1}(t, \mathbf{x}(t, \mathbf{z})) d t .
$$

If there exists an $a \in V$ with $\mathscr{F}(a)=0$ and $\operatorname{det}((\partial \mathscr{F} / \partial \mathbf{z})(a)) \neq 0$, then there exists a T-periodic solution $\varphi(t, \varepsilon)$ to system (25) such that $\varphi(0, \varepsilon) \rightarrow$ a as $\varepsilon \rightarrow 0$.

For the proof of Theorem 6, please see Corollary 1 of [21].

\section{Proof of Theorems and Corollaries}

The solution of system (1) with $\varepsilon=0$ such that $(x(0), y(0)$, $z(0))=\left(x_{0}, y_{0}, z_{0}\right)$ is

$$
\left(\begin{array}{l}
x(t) \\
y(t) \\
z(t)
\end{array}\right)=e^{A t}\left(\begin{array}{c}
x_{0} \\
y_{0} \\
z_{0}
\end{array}\right)+\int_{0}^{t} e^{A(t-s)}\left(\begin{array}{c}
h_{1}(s) \\
h_{2}(s) \\
h_{3}(s)
\end{array}\right) d s
$$

where

$$
A=\left(\begin{array}{ccc}
0 & -1 & 0 \\
1 & 0 & 0 \\
0 & 0 & a
\end{array}\right)
$$

So

$$
\left(\begin{array}{c}
x(t) \\
y(t) \\
z(t)
\end{array}\right)=\left(\begin{array}{c}
\cos (t) x_{0}-\sin (t) y_{0}+\int_{0}^{t}\left(\cos (t-s) h_{1}(s)-\sin (t-s) h_{2}(s)\right) d s \\
\sin (t) x_{0}+\cos (t) y_{0}+\int_{0}^{t}\left(\sin (t-s) h_{1}(s)+\cos (t-s) h_{2}(s)\right) d s \\
e^{a t} z_{0}+\int_{0}^{t} e^{a(t-s)} h_{3}(s) d s
\end{array}\right) .
$$


For studying the periodicity of this solution, we distinguish the two cases: $a=0$ and $a \neq 0$. These two cases will be studied, respectively, in Theorems 1 and 2.

3.1. Proof of Theorem 1. We will apply Theorem 6 to differential system (1) with $a=0$. We note that system (1) can be written as system (25) taking

$$
F_{0}(t, \mathbf{x})=\left(\begin{array}{c}
-y+h_{1}(t) \\
x+h_{2}(t) \\
h_{3}(t)
\end{array}\right)
$$

$$
F_{1}(t, \mathbf{x})=\left(\begin{array}{l}
P(x, y, z) \\
Q(x, y, z) \\
R(x, y, z)
\end{array}\right),
$$

$$
\mathbf{x}=\left(\begin{array}{l}
x \\
y \\
z
\end{array}\right), t=t
$$

We will study the periodic solutions of system (26) in our case, that is, the periodic solutions of system (1) with $a=0$ and $\varepsilon=0$. The solutions (32) with $a=0$ such that $(x(0), y(0), z(0))=\left(x_{0}, y_{0}, z_{0}\right)$ are

$$
\left(\begin{array}{l}
x(t) \\
y(t) \\
z(t)
\end{array}\right)=\left(\begin{array}{c}
\cos (t) x_{0}-\sin (t) y_{0}+\int_{0}^{t}\left(\cos (t-s) h_{1}(s)-\sin (t-s) h_{2}(s)\right) d s \\
\sin (t) x_{0}+\cos (t) y_{0}+\int_{0}^{t}\left(\sin (t-s) h_{1}(s)+\cos (t-s) h_{2}(s)\right) d s \\
z_{0}+\int_{0}^{t} h_{3}(s) d s
\end{array}\right) .
$$

These solutions are $2 \pi$-periodic if and only if

$$
\left(\begin{array}{l}
x(2 \pi) \\
y(2 \pi) \\
z(2 \pi)
\end{array}\right)=\left(\begin{array}{l}
x(0) \\
y(0) \\
z(0)
\end{array}\right) .
$$

We obtain the following periodicity conditions:

$$
\begin{array}{r}
\int_{0}^{2 \pi}\left(\cos (s) h_{1}(s)-\sin (s) h_{2}(s)\right) d s=0 \\
\int_{0}^{2 \pi}\left(-\sin (s) h_{1}(s)+\cos (s) h_{2}(s)\right) d s=0 \\
\int_{0}^{2 \pi} h_{3}(s) d s=0 .
\end{array}
$$

The set of periodic solutions (34) has dimension 3. To look for the periodic solutions of our system (1) with $a=0$, we must calculate the zeros $\mathbf{z}=\left(x_{0}, y_{0}, z_{0}\right)$ of the system $\mathscr{F}(\mathbf{z})=$ 0 , where $\mathscr{F}(\mathbf{z})$ is given by (29). The fundamental matrix $M(t)$ of the differential system (27) is

$$
M(t)=M_{\mathbf{z}}(t)=\left(\begin{array}{ccc}
\cos (t) & -\sin (t) & 0 \\
\sin (t) & \cos (t) & 0 \\
0 & 0 & 1
\end{array}\right) .
$$

Now computing the function $\mathscr{F}(\mathbf{z})$ given in (29), we find that the system $\mathscr{F}(\mathbf{z})=\mathbf{0}$ can be written as

$$
\left(\begin{array}{l}
\mathscr{F}_{1}\left(x_{0}, y_{0}, z_{0}\right) \\
\mathscr{F}_{2}\left(x_{0}, y_{0}, z_{0}\right) \\
\mathscr{F}_{3}\left(x_{0}, y_{0}, z_{0}\right)
\end{array}\right)=\left(\begin{array}{l}
0 \\
0 \\
0
\end{array}\right),
$$

where

$$
\begin{aligned}
& \mathscr{F}_{1}\left(x_{0}, y_{0}, z_{0}\right)=\frac{1}{2 \pi} \\
& \cdot \int_{0}^{2 \pi}(\cos (t) P(t, A(t), B(t), C(t)) \\
& \quad+\sin (t) Q(A(t), B(t), C(t))) d t, \\
& \mathscr{F}_{2}\left(x_{0}, y_{0}, z_{0}\right)=\frac{1}{2 \pi} \\
& \quad \cdot \int_{0}^{2 \pi}(-\sin (t) P(t, A(t), B(t), C(t)) \\
& \quad+\cos (t) Q(A(t), B(t), C(t))) d t, \\
& \mathscr{F}_{3}\left(x_{0}, y_{0}, z_{0}\right)=\frac{1}{2 \pi} \int_{0}^{2 \pi} R(t, A(t), B(t), C(t)) d t,
\end{aligned}
$$

where $A(t), B(t)$, and $C(t)$ have been defined in the statement of Theorem 1 . The zeros $\left(x_{0}^{*}, y_{0}^{*}, z_{0}^{*}\right)$ of the system

$$
\begin{aligned}
& \mathscr{F}_{1}\left(x_{0}, y_{0}, z_{0}\right)=0, \\
& \mathscr{F}_{2}\left(x_{0}, y_{0}, z_{0}\right)=0, \\
& \mathscr{F}_{3}\left(x_{0}, y_{0}, z_{0}\right)=0,
\end{aligned}
$$

with respect to the variables $x_{0}, y_{0}$, and $z_{0}$, provide periodic solutions of system (1) with $a=0$ and $\varepsilon \neq 0$ being sufficiently small if they are simple, that is, if

$$
\operatorname{det}\left(\left.\frac{\partial\left(\mathscr{F}_{1}, \mathscr{F}_{2}, \mathscr{F}_{3}\right)}{\partial\left(x_{0}, y_{0}, z_{0}\right)}\right|_{\left(x_{0}, y_{0}, z_{0}\right)=\left(x_{0}^{*}, y_{0}^{*}, z_{0}^{*}\right)}\right) \neq 0 .
$$


For simple zeros $\left(x_{0}^{*}, y_{0}^{*}, z_{0}^{*}\right)$ of system $(40)$, we obtain a $2 \pi$-periodic solution $\left(\begin{array}{c}x(t, \varepsilon) \\ y(t, \varepsilon) \\ z(t, \varepsilon)\end{array}\right)$ of differential system (1) with $a=0$, for $\varepsilon \neq 0$ being sufficiently small which tends to the periodic solution

$$
\left(\begin{array}{c}
x(t) \\
y(t) \\
z(t)
\end{array}\right)=\left(\begin{array}{c}
\cos (t) x_{0}^{*}-\sin (t) y_{0}^{*}+\int_{0}^{t}\left(\cos (t-s) h_{1}(s)-\sin (t-s) h_{2}(s)\right) d s \\
\sin (t) x_{0}^{*}+\cos (t) y_{0}^{*}+\int_{0}^{t}\left(\sin (t-s) h_{1}(s)+\cos (t-s) h_{2}(s)\right) d s \\
z_{0}^{*}+\int_{0}^{t} h_{3}(s) d s
\end{array}\right)
$$

of the differential system

$$
\begin{aligned}
& \dot{x}=-y+h_{1}(t), \\
& \dot{y}=x+h_{2}(t), \\
& \dot{z}=h_{3}(t),
\end{aligned}
$$

when $\varepsilon \rightarrow 0$.

This completes the proof of Theorem 1 .
3.2. Proof of Theorem 2. We will apply Theorem 5 to differential system (1) with $a \neq 0$. It can be written as system (25) taking

$$
\begin{aligned}
& F_{0}(t, \mathbf{x})=\left(\begin{array}{c}
-y+h_{1}(t) \\
x+h_{2}(t) \\
a z+h_{3}(t)
\end{array}\right), \\
& F_{1}(t, \mathbf{x})=\left(\begin{array}{c}
P(x, y, z) \\
Q(x, y, z) \\
R(x, y, z)
\end{array}\right), \quad \quad \mathbf{x}=\left(\begin{array}{c}
x \\
y \\
z
\end{array}\right), t=t .
\end{aligned}
$$

We will study the periodic solutions of system (26) in our case, that is, the periodic solutions of system (1) with $a \neq 0$ and $\varepsilon=0$. The solution (32), with $a \neq 0$ such that $(x(0), y(0), z(0))=\left(x_{0}, y_{0}, z_{0}\right)$, is

$$
\left(\begin{array}{l}
x(t) \\
y(t) \\
z(t)
\end{array}\right)=\left(\begin{array}{c}
\cos (t) x_{0}-\sin (t) y_{0}+\int_{0}^{t}\left(\cos (t-s) h_{1}(s)-\sin (t-s) h_{2}(s)\right) d s \\
\sin (t) x_{0}+\cos (t) y_{0}+\int_{0}^{t}\left(\sin (t-s) h_{1}(s)+\cos (t-s) h_{2}(s)\right) d s \\
e^{a t} z_{0}+\int_{0}^{t} e^{a(t-s)} h_{3}(s) d s
\end{array}\right) .
$$

These solutions are $2 \pi$-periodic if and only if

$$
\left(\begin{array}{l}
x(2 \pi) \\
y(2 \pi) \\
z(2 \pi)
\end{array}\right)=\left(\begin{array}{l}
x(0) \\
y(0) \\
z(0)
\end{array}\right) .
$$

We obtain the following periodicity conditions:

$$
\begin{aligned}
& \int_{0}^{2 \pi}\left(\cos (s) h_{1}(s)+\sin (s) h_{2}(s)\right) d s=0 . \\
& \int_{0}^{2 \pi}\left(-\sin (s) h_{1}(s)+\cos (s) h_{2}(s)\right) d s=0, \\
& z_{0}=\frac{1}{1-e^{2 \pi a}} \int_{0}^{2 \pi} e^{a(2 \pi-s)} h_{3}(s) d s .
\end{aligned}
$$


The set of the periodic solutions becomes

$$
\left(\begin{array}{c}
x(t) \\
y(t) \\
z(t)
\end{array}\right)\left(\begin{array}{c}
\cos (t) x_{0}-\sin (t) y_{0}+\int_{0}^{t}\left(\cos (t-s) h_{1}(s)-\sin (t-s) h_{2}(s)\right) d s \\
\sin (t) x_{0}+\cos (t) y_{0}+\int_{0}^{t}\left(\sin (t-s) h_{1}(s)+\cos (t-s) h_{2}(s)\right) d s \\
\frac{e^{a t}}{1-e^{2 \pi a}} \int_{0}^{2 \pi} e^{a(2 \pi-s)} h_{3}(s) d s+\int_{0}^{t} e^{a(t-s)} h_{3}(s) d s
\end{array}\right) .
$$

The set of the periodic solutions (48) has dimension two. To look for the periodic solutions of our system (1) with $a \neq 0$, we must calculate the zeros $\mathbf{z}=\left(x_{0}, y_{0}\right)$ of the system $\mathscr{F}(\mathbf{z})=$ 0 , where $\mathscr{F}(\mathbf{z})$ is given by (28). The fundamental matrix $M(t)$ of the differential system (27) is

$$
M(t)=M_{\mathbf{z}}(t)=\left(\begin{array}{ccc}
\cos (t) & -\sin (t) & 0 \\
\sin (t) & \cos (t) & 0 \\
0 & 0 & e^{a t}
\end{array}\right) .
$$

It verifies

$$
M^{-1}(0)-M^{-1}(2 \pi)=\left(\begin{array}{ccc}
0 & 0 & 0 \\
0 & 0 & 0 \\
0 & 0 & 1-e^{-2 \pi a}
\end{array}\right) .
$$

Consequently, all the assumptions of Theorem 5 are satisfied. Therefore, we must study the zeros of the system $\mathscr{F}(\mathbf{z})=0$ of two equations with two unknowns, where $\mathscr{F}$ is given in the statement of Theorem 5. More precisely, we have $\mathscr{F}(\mathbf{z})=\left(\begin{array}{c}\mathscr{F}_{1}\left(x_{0}, y_{0}\right) \\ \mathscr{F}_{2}\left(x_{0}, y_{0}\right)\end{array}\right)$ where

$$
\begin{aligned}
& \mathscr{F}_{1}\left(x_{0}, y_{0}\right)=\frac{1}{2 \pi} \int_{0}^{2 \pi}(\cos (t) P(A(t), B(t), C(t)) \\
& +\sin (t) Q(A(t), B(t), C(t))) d t
\end{aligned}
$$

$$
\begin{aligned}
& \mathscr{F}_{2}\left(x_{0}, y_{0}\right)=\frac{1}{2 \pi} \int_{0}^{2 \pi}(-\sin (t) P(A(\mathrm{t}), B(t), C(t)) \\
& +\cos (t) Q(A(t), B(t), C(t))) d t,
\end{aligned}
$$

where $A(t), B(t)$, and $C(t)$ have been defined in the statement of Theorem 2 . The zeros $\left(x_{0}^{*}, y_{0}^{*}\right)$ of the system

$$
\begin{aligned}
& \mathscr{F}_{1}\left(x_{0}, y_{0}\right)=0, \\
& \mathscr{F}_{2}\left(x_{0}, y_{0}\right)=0,
\end{aligned}
$$

with respect to the variables $x_{0}$ and $y_{0}$, provide periodic solutions of system (1) with $a \neq 0$ and $\varepsilon \neq 0$ being sufficiently small if they are simple, that is, if

$$
\operatorname{det}\left(\left.\frac{\partial\left(\mathscr{F}_{1}, \mathscr{F}_{2}\right)}{\partial\left(x_{0}, y_{0}\right)}\right|_{\left(x_{0}, y_{0}\right)=\left(x_{0}^{*}, y_{0}^{*}\right)}\right) \neq 0 .
$$

For simple zeros $\left(x_{0}^{*}, y_{0}^{*}\right)$ of system (52), we obtain a $2 \pi$ periodic solution $\left(\begin{array}{l}x(t, \varepsilon) \\ y(t, \varepsilon) \\ z(t, \varepsilon)\end{array}\right)$ of differential system (1) with $a \neq 0$, for $\varepsilon \neq 0$ being sufficiently small which tends to the periodic solution

$$
\left(\begin{array}{c}
x(t) \\
y(t) \\
z(t)
\end{array}\right)=\left(\begin{array}{c}
\cos (t) x_{0}^{*}-\sin (t) y_{0}^{*}+\int_{0}^{t}\left(\cos (t-s) h_{1}(s)-\sin (t-s) h_{2}(s)\right) d s \\
\sin (t) x_{0}^{*}+\cos (t) y_{0}^{*}+\int_{0}^{t}\left(\sin (t-s) h_{1}(s)+\cos (t-s) h_{2}(s)\right) d s \\
\frac{e^{a t}}{1-e^{2 \pi a}} \int_{0}^{2 \pi} e^{a(2 \pi-s)} h_{3}(s) d s+\int_{0}^{t} e^{a(t-s)} h_{3}(s) d s
\end{array}\right)
$$

of the differential system

$$
\begin{aligned}
& \dot{x}=-y+h_{1}(t), \\
& \dot{y}=x+h_{2}(t), \\
& \dot{z}=a z+h_{3}(t),
\end{aligned}
$$

when $\varepsilon \rightarrow 0$.

This completes the proof of Theorem 2.
3.3. Proof of Corollary 3. We must apply Theorem 1 with

$$
\begin{aligned}
\left(\begin{array}{l}
h_{1}(t) \\
h_{2}(t) \\
h_{3}(t)
\end{array}\right) & =\left(\begin{array}{c}
\sin (t) \\
\cos (t) \\
-\sin (t)
\end{array}\right), \\
\left(\begin{array}{l}
P(x, y, z) \\
Q(x, y, z) \\
R(x, y, z)
\end{array}\right) & =\left(\begin{array}{c}
x^{2}+y^{2}+x y z \\
y^{2}+z^{2}+x y z \\
x+y+z
\end{array}\right) .
\end{aligned}
$$


First, we can verify easily conditions (7)

$$
\begin{array}{r}
\int_{0}^{2 \pi}(\cos (s) \sin (s)-\sin (s) \cos (s)) d s=0 \\
\int_{0}^{2 \pi}\left(-\sin ^{2}(s)+\cos ^{2}(s)\right) d s=0 \\
\int_{0}^{2 \pi}-\sin (s) d s=0 .
\end{array}
$$

Computing the functions $\mathscr{F}_{1}, \mathscr{F}_{2}$, and $\mathscr{F}_{3}$ of Theorem 1 , we obtain

$$
\begin{aligned}
\mathscr{F}_{1}\left(x_{0}, y_{0}, z_{0}\right)= & \frac{1}{4} x_{0} y_{0}-\frac{1}{8} y_{0}^{2}+\frac{1}{8} x_{0}^{2}+\frac{1}{8} x_{0}-\frac{1}{8} y_{0}, \\
\mathscr{F}_{2}\left(x_{0}, y_{0}, z_{0}\right)= & -1+z_{0}+\frac{1}{4} x_{0} y_{0}+\frac{1}{8} y_{0}^{2}-\frac{1}{8} x_{0}^{2} \\
& -\frac{1}{8} x_{0}-\frac{1}{8} y_{0}, \\
\mathscr{F}_{3}\left(x_{0}, y_{0}, z_{0}\right)= & z_{0}-1 .
\end{aligned}
$$

The system $\mathscr{F}_{1}=\mathscr{F}_{2}=\mathscr{F}_{3}=0$ has four solutions $\left(x_{0}^{*}, y_{0}^{*}, z_{0}^{*}\right)$ given by $(0,0,1),(-1,0,1),(1 / 2, \sqrt{3} / 2,1)$, and $(1 / 2,-\sqrt{3} / 2,1)$.

Since

$$
\operatorname{det}\left(\left.\frac{\partial\left(\mathscr{F}_{1}, \mathscr{F}_{2}, \mathscr{F}_{3}\right)}{\partial\left(x_{0}, y_{0}, z_{0}\right)}\right|_{\left(x_{0}, y_{0}, z_{0}\right)=\left(x_{0}^{*}, y_{0}^{*}, z_{0}^{*}\right)}\right)
$$

for these four solutions $\left(x_{0}^{*}, y_{0}^{*}, z_{0}^{*}\right)$ is $-1 / 32,3 / 32,3 / 32$, and $3 / 32$, respectively, we obtain using Theorem 1 the solutions given in the statement of Corollary 3.

\subsection{Proof of Corollary 4. We must apply Theorem 2 with}

$$
\begin{aligned}
\left(\begin{array}{l}
h_{1}(t) \\
h_{2}(t) \\
h_{3}(t)
\end{array}\right) & =\left(\begin{array}{c}
\sin (t) \\
\cos (t) \\
\cos (t)
\end{array}\right), \\
\left(\begin{array}{l}
P(t, x, y, z) \\
Q(t, x, y, z) \\
R(t, x, y, z)
\end{array}\right) & =\left(\begin{array}{c}
x+y-x^{2} y \\
x+y-x y^{2} \\
x+y+z
\end{array}\right) .
\end{aligned}
$$

First, we can verify easily conditions (14):

$$
\begin{aligned}
\int_{0}^{2 \pi} 2 \cos (s) \sin (s) d s & =0 \\
\int_{0}^{2 \pi}\left(-\sin ^{2}(s)+\cos ^{2}(s)\right) d s & =0 \\
z_{0} & =\frac{-a}{a^{2}+1} .
\end{aligned}
$$

Computing functions $\mathscr{F}_{1}$ and $\mathscr{F}_{2}$ of Theorem 2, we obtain

$$
\begin{aligned}
& \mathscr{F}_{1}\left(x_{0}, y_{0}\right)=\frac{3}{4} x_{0} y_{0}+\frac{1}{2}+x_{0}+\frac{3}{8} y_{0}, \\
& \mathscr{F}_{2}\left(x_{0}, y_{0}\right)=-\frac{1}{2}-\frac{1}{8} x_{0}^{2}+\frac{5}{8} y_{0}^{2}-\frac{1}{8} x_{0}+y_{0} .
\end{aligned}
$$

The system $\mathscr{F}_{1}=\mathscr{F}_{2}=0$ has two solutions $\left(x_{0}^{*}, y_{0}^{*}\right)$ given by $(-1 / 2,-4 / 5+\sqrt{139} / 10),(-1 / 2,-4 / 5-\sqrt{139} / 10)$.

Since

$$
\operatorname{det}\left(\left.\frac{\partial\left(\mathscr{F}_{1}, \mathscr{F}_{2}\right)}{\partial\left(x_{0}, y_{0}\right)}\right|_{\left(x_{0}, y_{0}\right)=\left(x_{0}^{*}, y_{0}^{*}\right)}\right)
$$

for these two solutions is $(\sqrt{139} / 320)(16+3 \sqrt{139})$ and $(\sqrt{139} / 320)(-16+3 \sqrt{139})$ respectively, we obtain using Theorem 2 the solutions given in the statement of Corollary 4.

\section{Conflict of Interests}

The authors declare that there is no conflict of interests regarding the publication of this paper.

\section{References}

[1] D. Pi and X. Zhang, "Limit cycles of differential systems via averaging theory," Canadian Applied Mathematics Quarterly, vol. 17, no. 1, pp. 243-269, 2009.

[2] A. Cima, J. Llibre, and M. A. Teixeira, "Limit cycles of some polynomial differential systems in dimension 2,3 and 4 , via averaging theory," Applicable Analysis, vol. 87, no. 2, pp. 149-164, 2008.

[3] J. Llibre, J. Yu, and X. Zhang, "Limit cycles coming from the perturbation of 2-dimensional centers of vector fields in $\mathbb{R}^{3}$," Dynamic Systems and Applications, vol. 17, pp. 625-636, 2008.

[4] J. Llibre and A. Makhlouf, "Limit cycles of polynomial differential systems bifurcating from the periodic orbits of a linear differential system in $\mathbb{R}^{d}$, Bulletin des Sciences Mathematiques, vol. 133, no. 6, pp. 578-587, 2009.

[5] J. Llibre and A. Makhlouf, "Periodic orbits of a non-autonomous quadratic differential system obtained from third-order differential equations," Dynamical Systems, vol. 27, no. 2, pp. 161-168, 2012.

[6] J. Llibre, J. Yu, and X. Zhang, "Limit cycles for a class of thirdorder differential equations," The Rocky Mountain Journal of Mathematics, vol. 40, no. 2, pp. 581-594, 2010.

[7] F. S. Dias and L. F. Mello, "Nonlinear analysis of a quadratic system obtained from a scalar third order differential equation," Electronic Journal of Differential Equations, vol. 161, pp. 1-25, 2010.

[8] A. Makhlouf and M. Hamamda, "Limit cycles of third-order differential equations," Annals of Differential Equations, vol. 30, article 4, 2014.

[9] E. Esmailzadeh, M. Ghorashi, and B. Mehri, "Periodic behavior of a nonlinear dynamical system," Nonlinear Dynamics, vol. 7, no. 3, pp. 335-344, 1995.

[10] F. Minhós, "Periodic solutions for a third order differential equation under conditions on the potential," Portugaliae Mathematica, vol. 55, no. 4, pp. 475-484, 1998. 
[11] R. Reissig, "Periodic solutions of a third order nonlinear differential equation," Annali di Matematica Pura ed Applicata, vol. 92, pp. 193-198, 1972.

[12] A. U. Afuwape, "Remarks on Barbashin-Ezeilo problem on third-order nonlinear differential equations," Journal of Mathematical Analysis and Applications, vol. 317, no. 2, pp. 613-619, 2006.

[13] S. P. Hastings, "On the uniqueness and global asymptotic stability of periodic solutions for a third order system," Rocky Mountain Journal of Mathematics, vol. 7, no. 3, pp. 513-538, 1977.

[14] M. Bobieński and H. Żoładek, "Limit cycles for multidimensional vector fields. The elliptic case," Journal of Dynamical and Control Systems, vol. 9, no. 2, pp. 265-310, 2003.

[15] M. Bobieński and H. Żolądek, "Limit cycles of three-dimensional polynomial vector fields," Nonlinearity, vol. 18, no. 1, pp. 175-209, 2005.

[16] J. Llibre, D. D. Novaes, and M. A. Teixeira, "Higher order averaging theory for finding periodic solutions via Brouwer degree," Nonlinearity, vol. 27, no. 3, pp. 563-583, 2014.

[17] J. A. Sanders and F. Verhulst, Averaging Methods in Nonlinear Dynamical Systems, vol. 59 of Applied Mathematical Sciences, Springer, Berlin, Germany, 1985.

[18] F. Verhulst, Nonlinear Differential Equations and Dynamical Systems, Universitext, Springer, Berlin, Germany, 1996.

[19] I. G. Malkin, Some Problems of the Theory of Nonlinear Oscillations, Gosudarstvennoye Izdatel'stvo Tekhniko-Teoreticheskoy Literatury, Moscow, Russia, 1956, (Russian).

[20] M. Roseau, Vibrations non Linéeaires et Théorie de la Stabilité, vol. 8 of Springer Tracts in Natural Philosophy, Springer, New York, NY, USA, 1966, (French).

[21] A. Buică, J.-P. Françoise, and J. Llibre, "Periodic solutions of nonlinear periodic differential systems with a small parameter," Communications on Pure and Applied Analysis, vol. 6, no. 1, pp. 103-111, 2007. 


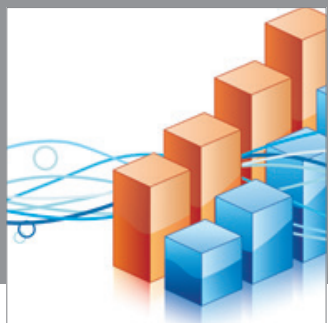

Advances in

Operations Research

mansans

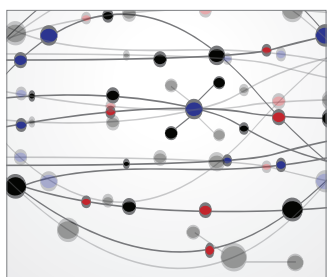

The Scientific World Journal
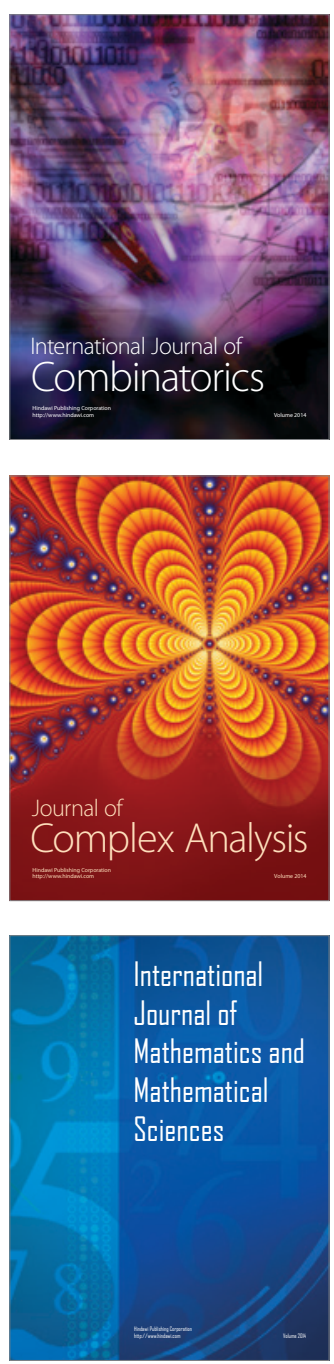
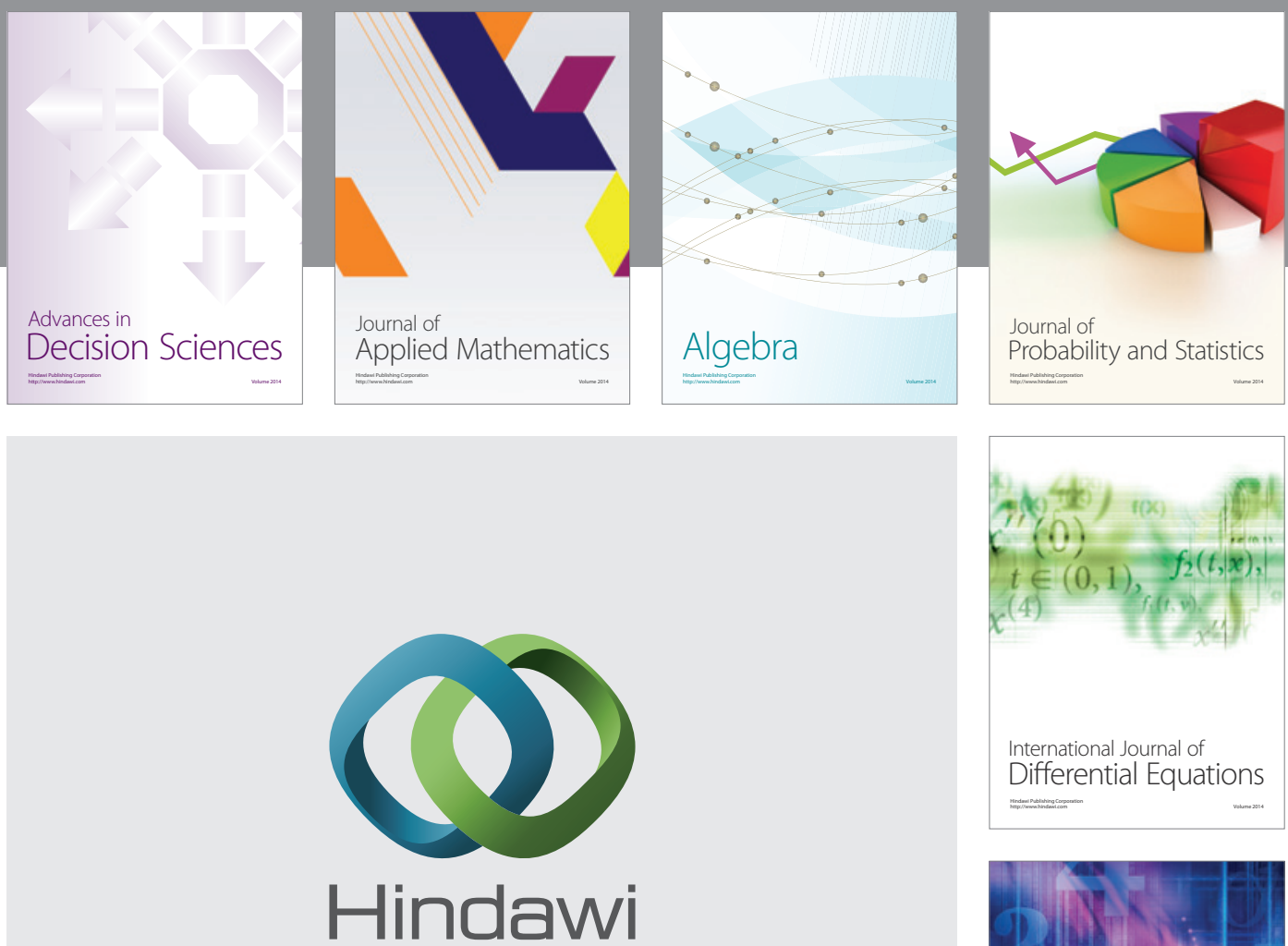

Submit your manuscripts at http://www.hindawi.com
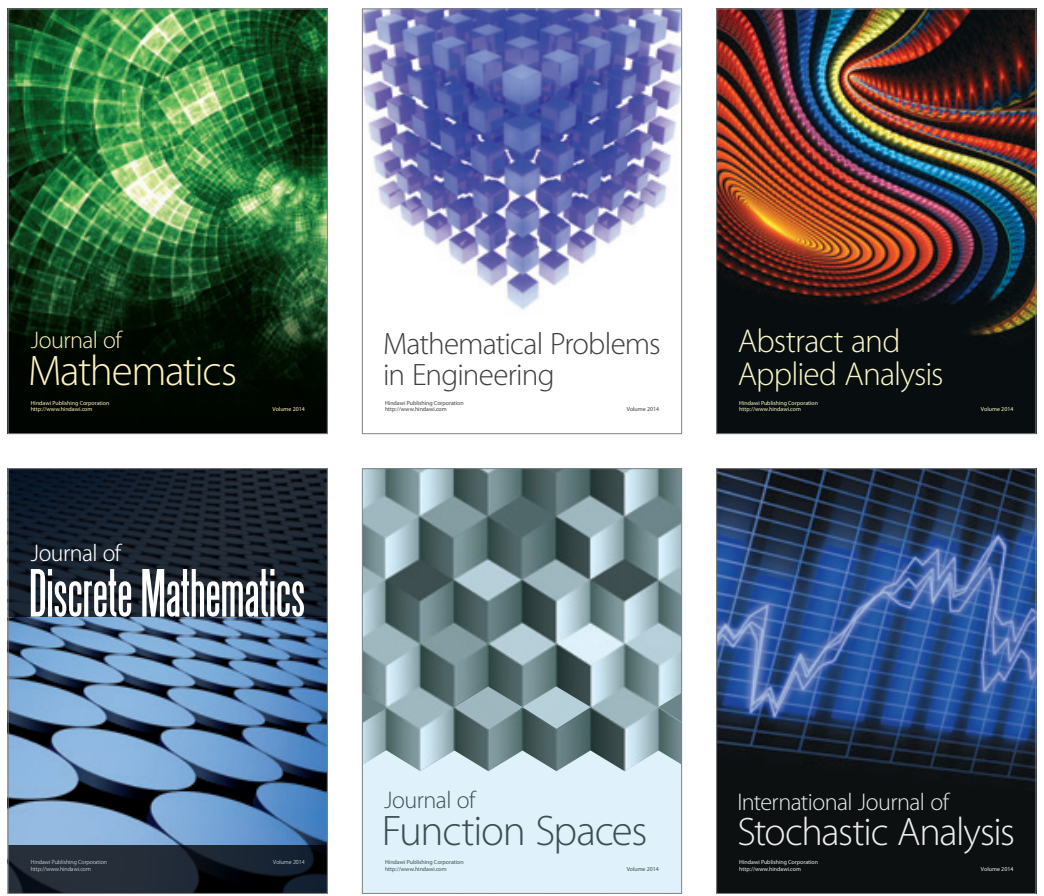

Journal of

Function Spaces

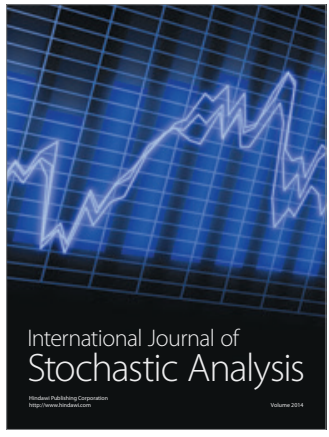

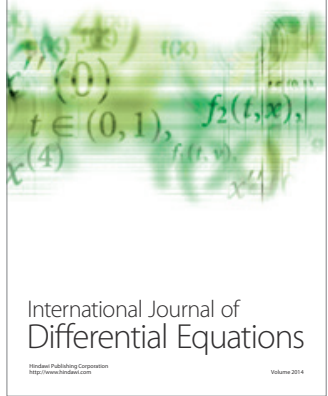
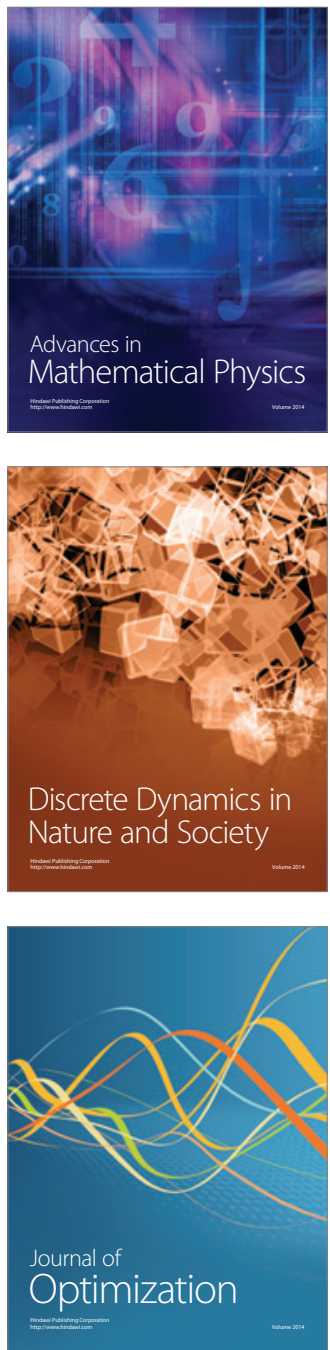